\title{
Gene expression disorders of innate antibacterial signaling pathway in pancreatic cancer patients: implications for leukocyte dysfunction and tumor progression
}

\author{
ROBERT SŁOTWIŃSKI I,2, ALEKSANDRA DĄBROWSKA², GUSTAW LECH ${ }^{3}$, \\ MACIEJ SEODKOWSKI 3 , SYLWIA M. SEOTWIŃSKA ${ }^{4}$ \\ ${ }^{1}$ Department of Surgical Research and Transplantology, Medical Research Center, Polish Academy of Sciences, Warsaw, Poland \\ ${ }^{2}$ Department of Immunology, Biochemistry and Nutrition, Medical University of Warsaw, Warsaw, Poland \\ ${ }^{3}$ Department of General, Gastroenterological and Oncological Surgery, Medical University of Warsaw, Warsaw, Poland \\ ${ }^{4}$ Department of Conservative Dentistry, Medical University of Warsaw, Warsaw, Poland
}

\begin{abstract}
The study was carried out to investigate changes in gene expression of innate antibacterial signaling pathways in patients with pancreatic cancer. Expression of the following genes was measured in peripheral blood leukocytes of 55 patients with pancreatic adenocarcinoma using real-time polymerase chain reaction (RT-PCR): TLR4, NOD1, MyD88, TRAF6 and HMGB1. The levels of expression of TLR4, NOD1 and TRAF6 genes were significantly elevated ( $p=0.007 ; p=0.001$ and $p=0.01$, respectively), while MyD88 expression was markedly reduced $(p=0.0002)$, as compared to controls. Expression of TLR4 and NOD1 exceeded the normal level more than 3.5-fold and there was a significant correlation found between the expression of these genes $(r=0.558, p<0.001)$. TLR4, NOD1 and MyD88 genes were expressed at a similar level both before and after surgery. No significant changes in the expression of HMGB1 gene were observed. The results of the study clearly indicate abnormal expression of genes belonging to innate antibacterial signaling pathways in peripheral blood leukocytes of patients with pancreatic cancer, which may lead to leukocyte dysfunction. Overexpression of TLR4, NOD1 and TRAF6 genes, and decreased MyD88 gene expression may contribute to chronic inflammation and tumor progression by up-regulation of the innate antibacterial response. The parameters tested are useful for monitoring innate immunity gene disorders and pancreatic cancer progression.
\end{abstract}

Key words: gene expression, innate immunity, pancreatic cancer.

(Centr Eur J Immunol 2014; 39 (4): 498-507)

\section{Introduction}

Pancreatic cancer ranks fourth in the causes of death from cancer, and the five-year survival rate ranges from $5 \%$ to $25 \%$ depending on the possibilities of surgery, which still remains the primary method of treatment $[1,2]$. One reason for the high mortality rate is the progress of the disease at diagnosis, which makes the radical surgical treatment effective only in $15-20 \%$ of patients [3]. Another problem is poor response to radiation and systemic therapy inhibiting the development of the disease and cancer relapse (e.g. adjuvant chemotherapy, immunotherapy) [4-6]. An important factor determining the rapid progression of cancer disease is high biological aggressiveness of the tumor contributing to the malnutrition of patients, tumor growth and formation of early metastasis.
Cancer-related malnutrition and immune disorders have been associated with poor prognosis, disease progression, treatment resistance and increased risk of postoperative complications, which may occur in up to $70-80 \%$ of patients [7-12]. As demonstrated in a number of studies, the improved outcomes of treatment are associated with a better understanding of the disorder mechanisms of the innate antibacterial immune response. This can be achieved by identification of specific gene profiles, which may promote chronic inflammation and pancreatic cancer progression, and can serve as new therapeutic targets [13-16]. Therefore, the tumor microenvironment is a complex problem and the cells infiltrating tumor play an extremely important role in the promotion of pancreatic cancer.

Correspondence: Prof. Robert Słotwiński, Department of Surgical Research and Transplantology, Medical Research Center, Polish Academy of Sciences, Warsaw, Poland, e-mail: robert_slotwinski@yahoo.com 
The discovery of Toll-like receptors (TLRs) has enabled a better understanding of disorders of the innate antibacterial response in patients with various diseases, in particular in patients with cancer. Toll-like receptors are a family of pattern-recognition receptors, which play a crucial role in the activation of innate and adaptive immunity, and can be expressed in several types of cells, such as macrophages, dendritic cells (DCs), B cells, T cells, monocytes and epithelial cells $[17,18]$. Toll-like receptor proteins recognize a large number of pathogen-associated molecular patterns such as bacterial lipopolysaccharides or viral RNA. All TLRs, with the exception of TLR3, signal via the MyD88 adapter protein (myeloid differentiation factor 88) [19, 20]. MyD88, TRAF6 [tumor necrosis factor $\alpha$ (TNF- $\alpha$ ) receptor-associated factor 6], TRIF (Toll/IL-1-receptor domain-containing adapter inducing interferon) and TRAM (TRIF-related adaptor molecule) proteins are key molecules in the cytoplasmic signaling cascade of the antibacterial response initiated by TLRs. TRAF6 is a member of the TNF receptor-associated factor family of proteins and is an E3 ubiquitin ligase, which catalyzes the synthesis of lysine polyubiquitin chain involved in the downstream activation of NF- $\kappa \mathrm{B}$ [21]. Toll-like receptor 4 -induced TAK1 autophosphorylation and activation require translocation of the MyD88-TRAF6-Ubcl3-cIAP-TAK1-IKK $\gamma$ signaling complex from TLR4 into the cytosol, which depends on TRAF6 and cIAPs [22]. TLR2 and TLR4 receptors were found to mediate the effects of HMGB1 (high mobility group box-1) in neutrophils and macrophages [23]. High-mobility group box-1 protein is an important protein binding to DNA, stabilizing nucleosomes and facilitating NF- $\kappa \mathrm{B}$ activation and gene transcription [24, 25]. High-mobility group box-1 modulates the inflammatory cascade in lipopolysaccharide (LPS)-activated macrophages by inducing the production of pro-inflammatory cytokines TNF- $\alpha$ and IL- $1 \beta$, while attenuating the release of anti-inflammatory mediators IL-10 and TGF- $\beta 1$ [26].

The abnormal expression of TLR receptors may be associated with sepsis, and autoimmune diseases (lupus erythematosus, rheumatoid arthritis, type I diabetes) [2731]. Interestingly, TLR receptors have been also detected in many tumor cell lines and tumors, including pancreatic ductal adenocarcinoma, and may be used as potential therapeutic targets [32, 33]. Toll-like receptors are involved in tumor cell proliferation, apoptosis and angiogenesis, while high expression of TLR4/MyD88 is correlated with poor prognosis in patients with colorectal cancer [34-39]. Lipopolysaccharide, a putative ligand of TLR4, may promote tumor progression by acting directly on cancer cells and increasing the invasive ability of pancreatic cancer cells through the TLR4/MyD88 signaling pathway [40, 41]. In contrast, the lipid A analogue (OM-174) with a dual stimulating effect on TLR2 and TLR4 receptors, induces regression of several tumor types in animal models [42-44]. Induction of MyD88 and TRIF, downstream
TLR pathways, perturbs cell cycle regulation in pancreatic cancer [45]. TRAF6 upregulates the expression of hypoxia-inducible factor (HIF-1 $\alpha$ ) and promotes tumor angiogenesis [46]. Toll-like receptor 4 in turn may be partly involved in the up-regulation of HIF-1 $\alpha$, and they both may synergistically promote the development of pancreatic adenocarcinoma [33].

Apart from TLRs, a significant role in the recognition of pathogen-related molecular patterns, and regulation of the innate antibacterial response is also played by other proteins belonging to the pathogen-associated molecular pattern (PAMP) receptors, including peptidoglycan recognition proteins, cytosolic NOD (nucleotide-binding oligomerization domain) proteins and TREM1 receptor (triggering receptor expressed on myeloid cells-1) [47]. Nucleotide-binding oligomerization domain-containing protein 1 is expressed in a wide variety of cell types of both hematopoietic and non-hematopoietic origin. They possess the ability to (e.g. NLRs - NOD-like receptors: NOD1, NOD2) to bind LPS and bacterial peptidoglycan as well as to transfer a signal independently of TLRs, which also results in NF- $\kappa \mathrm{B}$ activation and stimulates the expression of cytokine-coding genes (for NOD1: TNF- $\alpha$ and interleukin-6 (IL-6), and for NOD2: TNF- $\alpha$ and IL-1 $\beta$ ) and adhesive molecules [48]. Nucleotide-binding oligomerization domain-containing protein 1 recognizes the compounds of Gram-negative (meso-diaminopimelic acid with peptidoglycan) and some Gram-positive bacteria [49], whereas muramyl dipeptide is the ligand for NOD2, derived from the wall of Gram-positive and Gram-negative bacteria [50, 51]. NOD-like signaling is involved in the regulation of immune homeostasis in the gut. Nucleotide-binding oligomerization domain-containing protein 2 deficiency in mice can lead to a compromised epithelial barrier and impaired immune response to commensal microbiota [52]. In turn, NOD1 deficiency results in increased tumor formation associated with the inflammation of the colon in mice [53]. A recent study showed that NOD2 polymorphisms have been linked to gastric cancer that is induced by Helicobacter pylori infection [54].

Despite increasing evidence suggesting carcinogenic and therapeutic effects of stimulation or inhibition of TLRs, it still remains unclear what kind of changes in gene expression of TLR signaling pathways in leukocytes are associated with pancreatic cancer. The purpose of this study was to assess changes in the expression of TLR4, NOD1, MyD88, TRAF6 and HMGB1 genes in patients with pancreatic cancer.

\section{Material and methods}

Fifty-five patients (mean age $61.5 \pm 10.1$ ) with pancreatic cancer were subjected to pancreatic resection (pancreaticoduodenectomy or distal pancreatectomy with lymph node dissection) and included in the study. Histopatholog- 
ical examination for the whole group of patients detected adenocarcinoma in specimens resected. According to the TNM classification of the Union for International Cancer Control (UICC), 21 patients were in pathological stage I or II, while 34 patients were in stage III. The study did not include patients with inflammatory tumors, patients with organ transplants, patients treated with chemotherapy, radiotherapy or immunosuppressors, patients suffering from autoimmune diseases, patients with diabetes type 1 (insulin-dependent), chronic respiratory insufficiency (chronic obstructive pulmonary disease), cardiovascular insufficiency, kidney and liver diseases.

Disease-related malnutrition (undernutrition) has been detected in all patients (according to Nutritional Risk Screening, NRS 2002) and required an additional preoperative oral nutritional support (600-650 kcal per day, for 5-7 days). The mean preoperative weight loss within 3 months was $8.04 \pm 5.3 \%$ and $12.03 \pm 6.35 \%$ on day 7 after surgery. Body mass index (BMI) was within normal range. The mean BMI value for the entire group was 25.06 $\pm 4.86 \mathrm{~kg} / \mathrm{m}^{2}$ prior to surgery and $23.83 \pm 4.79 \mathrm{~kg} / \mathrm{m}^{2}$ on day 7 after pancreatic resection. The control group for molecular analyses comprised 20 healthy sex- and age-matched volunteers (mean age $59.3 \pm 9.4$ ).

\section{Sample collection and leukocyte isolation}

Blood samples were collected from a peripheral vein after admission to the surgical ward (day 0 before surgery) and on post-operative day 1. Leukocytes were isolated from $10 \mathrm{ml}$ of heparinized blood using Polymorphrep (AXIS-SHIELD PoC AS, Oslo, Norway). Briefly, blood was layered over Polymorphrep and centrifuged at room temperature $(500 \mathrm{~g} / 35 \mathrm{~min})$. The resulting fractions of mono- and polymorphonuclear leukocytes were harvested, washed in PBS solution to remove plasma and anticoagulant residues, suspended in a suitable medium and immediately frozen at $-70^{\circ} \mathrm{C}$, until used in further analysis.

\section{RNA extraction and reverse transcription}

Total RNA was extracted from leukocytes using a NucleoSpin Total RNA, DNA and Protein Isolation kit
(Macherey-Nagel GmbH \& Co. Dueren, Germany), according to manufacturer's instructions and restrictive procedure for RNA degradation. Isolated RNA was evaluated qualitatively and quantitatively using a NanoDrop ND1000 spectrophotometer (Thermo Fisher Scientific, Wilmington, DE, USA). RNA sample was considered pure when the $\mathrm{A}_{260} / \mathrm{A}_{280}$ ratio was in the range 1.7-2.0. Then the firststrand cDNA was synthesized using a VerteKit reverse transcription kit (Novazym, Poznań, Poland). Briefly, $5 \mu \mathrm{g}$ of total RNA was incubated at $70^{\circ} \mathrm{C}$ for 5 minutes with oligo(dT) ${ }_{15}$ primers. Afterwards, M-MLV buffer, dNTPs mix and Moloney Murine Leukemia Virus reverse transcriptase were added and the mix was incubated at $37^{\circ} \mathrm{C}$ for 60 minutes. Reaction was terminated by $10 \mathrm{~min}-$ utes incubation at $70^{\circ} \mathrm{C}$. The reverse transcription products were analyzed again on a NanoDrop spectrophotometer.

\section{Real-time PCR}

Real-time polymerase chain reaction (RT-PCR) was performed using a LightCycler 2.0 Instrument and LightCycler $^{\circledR}$ FastStart DNA Master SYBR Green I detection kit (Roche Applied Science, Basel, Switzerland Cat. No. 12239264001 ) as per the manufacturer's protocol. The RT-PCR reaction was based on primers specific for the genes tested, designed in the NCBI/Primer-BLAST application. The mixture contained $2 \mu \mathrm{l}$ of reverse transcription product, $2 \mu \mathrm{l}$ of primers, $0.6 \mu \mathrm{l}$ of $\mathrm{MgCl}_{2}$ and $2 \mu \mathrm{l}$ of LightCycler $^{\circledR}$ FastStart DNA Master SYBR Green I. DNaseRNase-free distilled water was added to a total volume of $20 \mu \mathrm{l}$ per capillary. The PCR reaction was run after pre-incubation at $95^{\circ} \mathrm{C}$ for $10 \mathrm{~min}$, followed by 45 denaturation cycles at $95^{\circ} \mathrm{C}$ for 15 second, annealing for 10 seconds at optimized temperature (Table 1) and elongation at $72^{\circ} \mathrm{C}$ for 15 seconds. After 45 cycles, a melting curve was generated by slowly increasing $\left(0.2^{\circ} \mathrm{C} / \mathrm{s}\right)$ the temperature from $70^{\circ} \mathrm{C}$ to $99^{\circ} \mathrm{C}$ and measuring the fluorescence. In each assay, the level of GAPDH mRNA as well as TLR4, NOD1, MyD88, TRAF6 and HMGB1 mRNA were measured simultaneously under identical conditions. The primer sequences and annealing temperatures are summarized in Table 1.

Table 1. Primer sequences and appropriate annealing temperatures used during real-time PCR reaction

\begin{tabular}{llll}
\hline Gene & Forward sequence & Reverse sequence & $\begin{array}{l}\text { Temperature } \\
\text { of annealing }\end{array}$ \\
\hline TLR4 & 5'-GCCCTGCGTGGAGGTGGTTC-3' & 5'-GTCCAGAAAAGGCTCCCAGGGC-3' & $61^{\circ} \mathrm{C}$ \\
\hline MyD88 & 5'-GATTGTAGGGCAGGGCGGCG-3' & 5'-AGCAGCCAGGGGAAGGGAGG-3' & $61^{\circ} \mathrm{C}$ \\
\hline TRAF6 & 5'-AGGGACCCAGCTTTCTTTGTGTGC-3' & 5'-CCGTGCGTGGCAGTTCCACC-3' & $62^{\circ} \mathrm{C}$ \\
\hline NOD1 & 5'-GCCCCGCGTTCAGGTCGAAA-3' & 5'-GTGAGGCGGCTGAAGCAGGG-3' & $61^{\circ} \mathrm{C}$ \\
\hline HMGB1 & 5'-GGCAAGTGAGAGCCGGACG-3' & 5'-GCTTCTTCTTATGCTCCTCCCGACA-3' & $63 / 62^{\circ} \mathrm{C}$ \\
\hline GAPDH & 5'-GTGAAGCAGGCGTCGGAGGG-3' & 5'-GCTCTTGCTGGGGCTGGTGG-3' & $61^{\circ} \mathrm{C}$ and $62^{\circ} \mathrm{C}$ \\
\hline
\end{tabular}


The results were analyzed using the method of double delta, assuming that the expression of the gene in the treated groups is $2^{-\Delta \Delta \mathrm{Ct}}$, where $\Delta \Delta \mathrm{Ct}=[\mathrm{Ct}$ target gene $-\mathrm{Ct}$ $\mathrm{GAPDH}]$ is the treated sample, and [Ct target gene $-\mathrm{Ct}$ GAPDH] is the control sample [55]. Each experiment was carried out in triplicate using GAPDH as an internal standard. This calculation formula shows the expression of the gene in the test group as multiples of the control group expression (expression of the gene in the healthy subject equals 1 ). Results are presented as mean $\pm \mathrm{SD}$.

\section{Ethics}

Patients gave written consent after details of the protocol were fully explained to them. The study protocol was approved by the Medical University Ethics Committee and conforms to the ethical guidelines of the World Medical Association Declaration of Helsinki.

\section{Statistical analysis}

Statistical analysis was performed using Statsoft Statistica v.9.0 software. Mann-Whitney $U$ test or Wilcoxon's signed-rank test with the Bonferroni correction was used to evaluate the statistical significance between the gene expression in pancreatic cancer patients and healthy volunteers, and to compare the level of expression before and after surgery. Spearman's rho test was used to assess the relationship between the expression of the genes. Differences at $p<0.05$ were considered to be statistically significant.

\section{Results}

\section{Gene expression before and after surgery}

The peripheral blood leukocytes of patients with pancreatic cancer had significantly elevated expression of genes encoding receptor proteins TLR4 and NOD1. The expression of these genes was significantly increased, compared

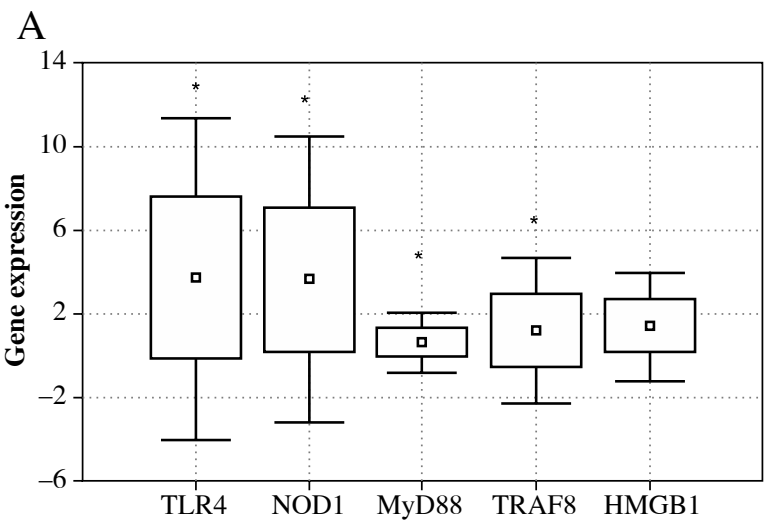

with healthy subjects, both before and after surgery (preoperative TLR $4 p=0.007$, and postoperative $p=0.002$, while for NOD1 $p=0.001$ and $p=0.003$, respectively) (Fig. 1). The levels of expression of TLR4 and NOD1 exceeded the norm more than 3.5-fold. Expression of genes coding for NOD1 and TLR4 protein receptors was also significantly increased compared to the expression of genes encoding the remaining proteins, i.e., MyD88, TRAF6 and HMGB1 ( $p<0.05$ for all values).

Expression of the gene encoding the intracellular MyD88 protein, prior to surgery (day $0, p=0.0001$ ) and day 1 after surgery ( $p=0.00003$ ) was significantly reduced when compared with the norm. TRAF6 gene expression prior to surgery was markedly higher compared to healthy subjects $(p=0.01)$, whereas after surgery it did not differ significantly from the norm. The study showed no significant deviation from the normal gene expression of the HMGB1 protein, both before and after surgery. Extensive surgical trauma had no statistically significant effect on the changes in the expression of any of the genes tested. Interestingly, a positive correlation was found in patients, who expressed the study genes in parallel between the expression of NOD1 and TLR4 genes (before surgery $r=0.558, p<0.001$, after surgery $r=0.466, p<0.001$ ) (Fig. 2). Although the expression of other genes was correlated and statistically significant, it was considerably lower (data not shown).

\section{Discussion}

Chronic inflammation and activation of the innate immune effectors creates favorable conditions for the tumor progression. Toll-like receptor-mediated changes in gene expression of the innate antibacterial signaling pathway in patients with pancreatic cancer are an important element in the diagnosis of immunity disorders associated with high biological aggressiveness of the tumor and disease pro-

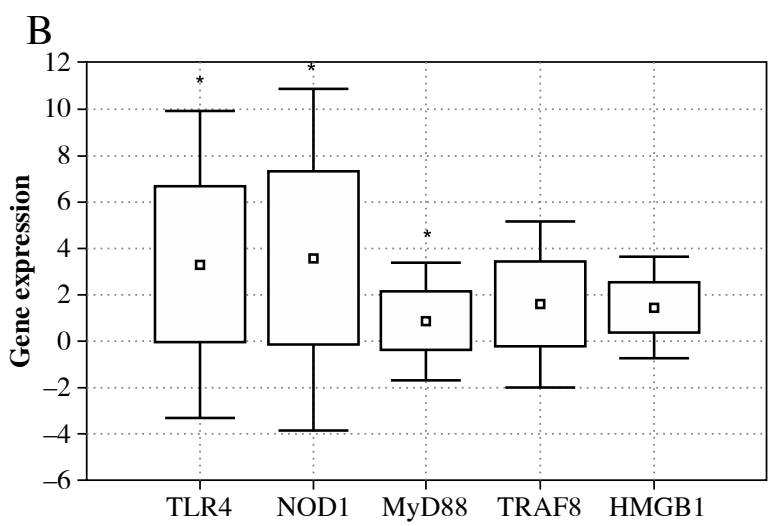

Fig. 1. Expression of genes encoding TLR4, NOD1, MyD88, TRAF6 and HMGB1 proteins in peripheral blood leukocytes of patients with pancreatic cancer before $(\mathbf{A})$ and after surgery $(\mathbf{B})$ in comparison with the control group $(* p<0.05)$ 


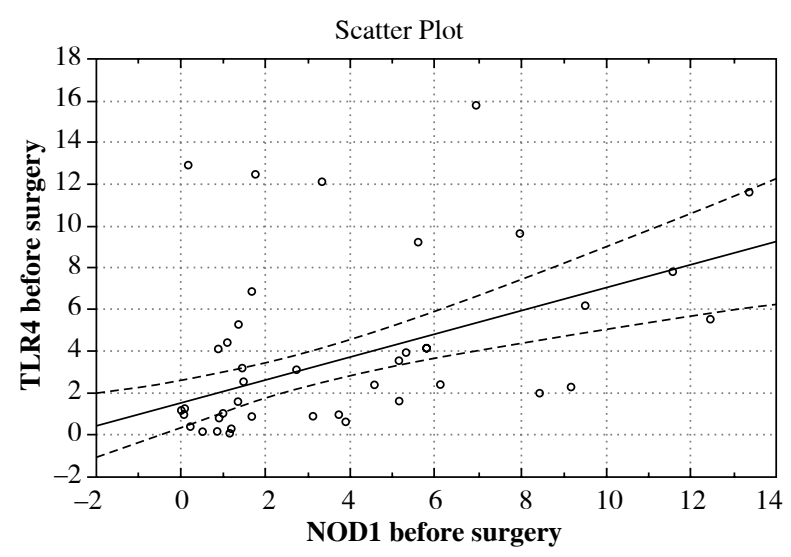

Fig. 2. The correlation between the expression of TLR4 and NOD1 genes in the peripheral blood leukocytes of patients with pancreatic cancer (pre-operative period)

gression. Disorders of gene expression in peripheral blood leukocytes that infiltrate tumor cells have a major impact on the course of cancer disease by supporting chronic inflammation in the tumor microenvironment. Local antibacterial TLR-related response may enhance the activity of cancer cells and promote tumor progression [40, 41], but may also induce regression of the tumor [44]. Most studies have evaluated the expression of TLRs in tumor tissue while paying less attention to the gene expression changes in the TLR signaling pathway in peripheral blood leukocytes that may reflect the impact of cancer disease such as malnutrition associated with a high metabolic activity of the tumor. The majority of our patients were diagnosed with advanced pancreatic tumor and all of them were malnourished and required additional preoperative oral nutritional support. This study presents the disturbances of the gene expression profiles responsible for the innate immunity of peripheral blood leukocytes that can be discussed based on the previous studies concerning the expression of proteins belonging to the TLR signaling pathway in blood leukocytes and pancreatic tumor tissue. However, we have not found in the available literature any reports referring to the gene expression changes in the TLR signaling pathway in peripheral blood leukocytes of pancreatic cancer patients.

The results of our study clearly indicate a significantly increased expression of genes (examined at the mRNA level) encoding TLR4, NOD1 receptor and TRAF6 signaling protein, and reduced MyD88 gene expression in peripheral blood leukocytes. Expression of TLR4 and NOD1 genes exceeded the normal level more than 3.5-fold. These findings suggest that the disorders of innate immunity gene expression, through various mediators, may cause dysfunction of the leukocyte immune response in malnourished pancreatic cancer patients, resulting in disease progression.
The increased expression of TLR4 and NOD1 genes and reduced expression of MyD88 gene remained at a similar level both before and after surgery, which may suggest a "stable genetic defect" in the leukocytes of patients with pancreatic cancer. Worsening malnutrition after major surgery also had no significant effect on the expression of the genes tested. Moreover, the expression of the NOD1 and TLR4 genes was significantly elevated when compared to the expression of other genes investigated. In addition, there was a significant correlation found between the NOD1 and TLR4 gene overexpression. These findings indicate the importance of TLR4 and NOD1 signaling pathways in the innate immune response of pancreatic cancer patients and tumor development. The increased expression of genes coding for TLR4 and NOD1 proteins is an important diagnostic tool and should be used for monitoring therapeutic intervention in patients with pancreatic ductal adenocarcinoma. As shown by other studies, TLR4 and TLR7 receptors are overexpressed on leukocytes in the pancreatic tumor microenvironment and by pancreatic cancer cells. Additionally, TLR ligands have been shown to accelerate pancreatic carcinogenesis in mice, while TLR blockade delayed tumor progression $[15,16]$. The present study has demonstrated that overexpression of TLR4 and NOD1 genes in leukocytes of pancreatic cancer patients can cause the enhancement of response to LPS and other bacterial wall proteins (e.g. compounds of Gram-negative bacteria, bacterial peptidoglycan). This in turn increases transfer of a signal independently via TLR4 or NOD1 receptors, possibly resulting in the activation of NF- $\kappa B$ and induction of the expression of cytokine coding genes, leading to chronic local inflammation and tumor progression. More specifically, we hypothesize that the elevated expression of the above genes caused by the higher level of TLR4 and NOD1 receptors increases the sensitivity of leukocytes to bacterial antigens. Lipopolysaccharide (LPS) is a well-established agonist of TLR4 and could be a triggering factor in the initiation and progression of pancreatic cancer [56, 57]. Toll-like receptor 4 expression in monocytes of healthy controls can be up-regulated by LPS and this has been confirmed by previous studies [58]. Thus, it is suggested that the leukocytes of patients with pancreatic cancer can be sensitized to LPS (by TLR4 and NOD1 gene overexpression), which sustains chronic inflammation. Confirmation of this hypothesis requires further investigations. Signaling pathway analyses in the genome-wide association study (data from 3,851 of pancreatic cancer cases) revealed that the NOD1 gene was associated with a risk of pancreatic cancer $(H$. pylori - cytokine signaling pathway) [59]. Previous studies have also found increased expression of TLR2 and TLR4 receptors on monocytes in patients with sepsis, and after trauma [28, 60, 61]. In the last study, the expression of TLR4 and TLR2 receptors on leukocytes increased during $16 \mathrm{~h}$ of incubation with LPS, but remained unchanged in healthy controls. Armstrong 
et al. [62] showed a differential expression of TLR2 and TLR4 on monocytes in human sepsis. TLR4 mRNA was significantly increased in septic patients, while there was no corresponding increase in the TLR4 protein. The results of these studies indicate a relationship between the innate immune response in the pancreatic cancer and response to infection or trauma in patients without cancer disease.

Another interesting finding of our study is a lower expression of the MyD88 gene encoding an adaptor protein, which is a key signaling protein for numerous extracellular or endosomal TLRs. In the literature, there are no data on the MyD88 gene expression in leukocytes of patients with pancreatic cancer. A high expression of TLR4 and MyD88 in tissue samples of colorectal cancer is associated with liver metastases and is an independent predictor of poor prognosis [37]. The results of the presented studies suggest that a decreased expression of MyD88 can facilitate the development of pancreatic cancer and should be considered important in the monitoring of disease progression. It should be emphasized that synthetic inhibitors of MyD88 pathway were shown to exacerbate pancreatic fibro-inflammation and accelerate carcinogenesis [45], while mice deprived of TLR2 and the MyD88 adaptor protein were more susceptible to infections [63]. The reason for the reduced gene expression of MyD88 is difficult to explain, but it is undoubtedly an alarming symptom associated with the development of pancreatic cancer. The inflammatory response to LPS in mice deprived of MyD88 protein was significantly reduced but still present, which suggests cooperation of other signaling cascades in the antibacterial response [64]. Signal transduction through NOD-like receptors (NLRs) does not require any participation of the MyD88 protein. Pancreatic cancer may be one of the direct causes of a significantly lower MyD88 expression in our patients with advanced tumor, which could indicate the superiority of stimuli reaching the NOD1 receptor. Silencing the expression of MyD88 gene in peripheral blood leukocytes completely reversed the effects of LPS by activation of new signal pathways. This mechanism probably can create favorable conditions for the progression of pancreatic cancer and allow tumor cells to evade the immune response. The precise cause of the abrogation of MyD88 expression requires further studies. Other authors have shown that TLR4 is overexpressed in human pancreatic ductal adenocarcinoma (in $69-76 \%$ of the analyzed tumors), and inhibition of MyD88-dependent pathways clearly accelerated the development of pancreatic tumor and could induce the formation of aggressive cancer cells [16, 33, 65]. According to these findings, upon MyD88 blockage, DCs seem to induce the formation of pancreatic antigen-restricted Th2-deviated CD4+ T cells. In this study, stromal leukocytes from patients showed an increased TLR4 expression, and such an up-regulation has been also reported both in the epithelial and stromal cells in KRAS-mutated mice. Interestingly, inhibition of TLR4 in these mice had a protective effect against tumorigenesis (slower tumor growth). In our opinion, the adjuvant therapy of pancreatic cancer should also focus on the regulation of gene expression in peripheral blood leukocytes. Hypothetically, the influx and recruitment of leukocytes to the tumor microenvironment with the formation of inflammatory infiltration may facilitate cancer cell escape from the control of the immune system in poorly defined mechanisms, e.g. chronic inflammation and immune suppression in the tumor microenvironment with immune responses mediated by Treg, dendritic cells, MDSCs (myeloid-derived suppressor cells) and Th17 cells; MMPs-matrix metalloproteinases and HIF- $\alpha$-hypoxia-inducible transcription factor overexpression or increased production of IL-10 and $\mathrm{H}_{2} \mathrm{O}_{2}$. For example, pancreatic cancer shows a significant increase in the infiltration of macrophages, and tumor-associated macrophages developing from peripheral blood monocytes (M2 subtype) exert an immunosuppressive effect and are associated with poor prognosis. Similarly, the M1 subtype macrophages promote tumor growth mediated by the TNF- $\alpha$ production $[66,67]$. In addition, neutrophil infiltrations are reported in pancreatic adenocarcinomas and have been associated with the undifferentiated types of carcinoma and poor prognosis [68].

Changing the microenvironment of the tumor cells by regulating the expression of genes encoding proteins of the innate antibacterial signaling pathway in leukocytes infiltrating tumor cells is a novel therapeutic approach. This applies to both the main tumor mass, which not always can be removed surgically, and micrometastases that are beyond the reach of diagnostic tests. Furthermore, the assessment of the gene expression profile in leukocytes can be useful for monitoring the course of disease and genetic effectiveness of the treatment. Our previous study in a small group of malnourished pancreatic cancer patients with overexpression of TLR4 gene in peripheral blood leukocytes revealed that enteral supplementation of glutamine (diet consisting of $20 \mathrm{~g}$ glutamine per day) may decrease TLR4 expression within five days prior to surgery [69]. This surprising effect of immunonutrition in pancreatic cancer patients could be associated with a decrease in the amount of stimulants reaching the TLR4 receptor (e.g. by blocking LPS or DAMPs - damage-associated molecular pattern molecules, interacting with TLR4 receptor). An experimental study showed that the enteral administration of glutamine reduced the elevated expression of TLR4, signal adaptor protein MyD88, and TRAF6 mRNA in the intestinal mucosa in response to LPS-induced endotoxemia in rats [70]. Investigations of the effect of this treatment on the number of infectious complications and course of the disease are in progress. It is also known that fatty acids (Omega-3) can have a selective inhibitory effect on the signaling cascade related to the innate antibacterial response by blocking the interaction of endotoxin with the TLR4 receptor and intracellular signaling proteins [71]. 
The concept of modulation (mainly inhibition) of the signaling pathway associated with TLR4 in pancreatic cancer has been already emphasized in other studies. An in vitro study revealed that the invasive capacity of human pancreatic cancer cells is based on the TLR4-dependent $\mathrm{NF}-\kappa \mathrm{B}$ activation [41]. It has been demonstrated that LPS can activate NF- $\kappa \mathrm{B}$ in pancreatic cancer cells via TLR4/ MyD88 pathway. Nuclear factor $\kappa B$ is one of the key factors linking inflammation with cancer progression. Another study correlated the level of TLR4 expression with tumor size, lymph node involvement, venous invasion and pathological stage [33]. Patients with TLR4 overexpression had significantly shorter survival [72]. Most studies have shown that the increased expression of TLR4 is not a disorder limited to pancreatic cancer. Toll-like receptors (TLRs) are involved in the development of infectious diseases, autoimmune and neurodegenerative diseases and cancer [73]. Toll-like receptor 4 expression has been also detected in head and neck cancer, breast cancer, lung cancer, gastric cancer, hepatocellular carcinoma, colorectal cancer, ovarian cancer, cervical cancer, prostate cancer and melanoma [33, 35]. The results of these studies indicate that the evaluation of TLR4 expression does not differentiate cancerous tumors, which makes diagnosis and treatment difficult. Probably assessments of genetic profile differences between these patients will be more helpful.

TRAF6 gene overexpression revealed in our study may confirm the participation of this gene in the development of pancreatic cancer. Experimental studies by other authors have shown that TRAF6 is an oncogene, which is important for RAS-mediated oncogenesis and provide a mechanistic explanation for the already apparent importance of constitutive NF- $\kappa \mathrm{B}$ activation in the RAS-driven lung cancer [74]. The participation of the TRAF6 gene in the pathogenesis and progression of pancreatic cancer is not clear. TRAF6 as a part of signaling complex (included MyD88IRAK4-IRAK1-TRAF6) is required for the pro-inflammatory cytokine production induced by TLR7 and TLR9 signaling. The increased expression of the TRAF6 gene may suggest that in addition to TLR4 and NOD1 overexpression, other signaling receptors (e.g. TLR7 or TLR9) take part in the innate immunity response of pancreatic cancer patients [15]. Toll-like receptor 7 was found to be highly expressed both in inflammatory (in $40-65 \%$ of cells) and cancer cells. Recently, TLR9 has been linked to acute pancreatitis and cancer [75]. According to these studies, potential inhibitors of the TLR signaling pathway and growth of pancreatic cancer cells are under research [76]. On the other hand, TLR2/4/9 agonists seem to be good candidate molecules prolonging survival in patients with different types of cancer by inducing the expression of TNF- $\alpha$ and inducible nitric oxide synthase (iNOS), activating dendritic cell trafficking and its associated tumor-specific cytotoxic T-cell responses [77-80]. The therapeutic mechanism of
TLR7 agonist (Imiquimod) is associated with the activation of immune cells involved in an effective antitumor immune response (B lymphocytes, plasma cells, NK, Langerhans cells) [81]. Thus, TLRs have a dual role, (i) when used as a target for immunostimulation, and (ii) when used directly to kill cancer cells.

The normal expression of the gene coding for the HMGB1 protein in the present study was rather surprising. This could be due to the lack of energy substrates necessary for the expected increased expression of this gene. HMGB1 is one of the most characteristic genes deregulated in solid tumor tissue and serum, including pancreatic cancer [82, 83]. The serum level of HMGB1 tends to increase with the progression of gastric and pancreatic cancers and is a powerful diagnostic biomarker for pancreatic ductal adenocarcinoma [82, 84]. The HMGB1/RAGE (receptor for advanced glycation endproducts) inflammatory pathway promotes pancreatic tumor growth by regulating mitochondrial bioenergetics [85]. Lack of RAGE or inhibition of HMGB1 release, diminished production of ATP and slowed tumor growth in vitro and in vivo.

In summary, the results of our study clearly indicate an abnormal gene expression in the innate antibacterial signaling pathways in peripheral blood leukocytes of patients with pancreatic cancer, which may lead to leukocyte dysfunction. Overexpression of TLR4, NOD1 and TRAF6 genes, and a decreased MyD88 gene expression may contribute to chronic inflammation and tumor progression by up-regulation of the innate antibacterial response (e.g. to LPS). We postulate that the pathologic gene expression of receptors and proteins regulating innate antibacterial response to various stimuli must be primarily included in the analysis of a multistep sequence of events leading to the development and progression of pancreatic cancer. Analysis of gene expression included in the current study in peripheral blood leukocytes can be useful for the diagnosis of gene disorders in the innate antibacterial signaling pathways and should be important in the monitoring of disease progression and therapy efficacy. TLR4 gene overexpression in peripheral blood leukocytes, and TLR4 receptor overexpression detected by other researchers in pancreatic cancer cells, suggest a direct link between the peripheral and local innate immune disorders.

The results presented show that in order to improve outcomes of the therapy in the group of patients with pancreatic cancer, more attention should be focused on the explanation of genetic mechanisms regulating the innate antibacterial response. Attempts to modulate the innate antibacterial immune response in pancreatic cancer patients, e.g. by blocking the expression of genes of selected signaling pathways (e.g. TLR4 gene inhibition), are promising and indicate that in the future it may be a valuable way assisting the primary therapy. 
This study was supported by the Ministry of Science and Higher Education, Poland (grant no. 2 PO5B 05928 and $3068 \mathrm{~B}$ P01). The authors declare no conflict of interest.

\section{References}

1. Vincent A, Herman J, Schulick R, et al. (2011): Pancreatic cancer. Lancet 378: 607-620.

2. Olson SH, Kurtz RC (2013): Epidemiology of pancreatic cancer and the role of family history. J Surg Oncol 107: 1-7.

3. Real FX (2003): A catastrophic hypothesis for pancreas cancer progression. Gastroenterology 124: 1958-1964.

4. Goodman KA, Hajj C (2013): Role of radiation therapy in the management of pancreatic cancer. J Surg Oncol 107: 86-96.

5. Ghaneh P, Costello E, Neoptolemos JP (2007): Biology and management of pancreatic cancer. Gut 56: 1134-1152.

6. Ben-Josef E, Lawrence TS (2008): Chemioradiotherapy for unresectable pancreatic cancer. Int J Clin Oncol 13: 121-126.

7. Lowe SW, Bodis S, McClatchey A, et al. (1994): p53 status and efficacy of cancer therapy in vitro. Science 266: 807-810.

8. Harada T, Okita K, Shiraishi K, et al. (2002): Interglandular cytogenetic heterogeneity detected by comparative genomic hybridization in pancreatic cancer. Cancer Res 62: 835-839.

9. Xiong HQ (2004): Molecular targeting therapy for pancreatic cancer. Cancer Chemothr Pharmacol 54 (suppl 1): 69-77.

10. Ueda S, Ogata S, Tsuda H, et al. (2004): The correlation between cytoplasmic overexpression of epidermal growth factor receptor and tumor aggressiveness: Poor prognosis in patients with pancreatic ductal adenocarcinoma. Pancreas 29: 1-8.

11. Li Y, VandenBoom TG 2nd, Kong D, et al. (2009): Up-regulation of miR-200 and let-7 by natural agents lead to the reversal of epithelial-mesenchymal transition in gemcitabine-resistant pancreatic cancer cells. Cancer Res 69: 67046712.

12. DeOliveira ML, Winter JM, Schafer M, et al. (2006): Assessment of complications after pancreatic surgery. Ann Surg 244: 931-939.

13. Vaz J, Andersson R (2014): Intervention on toll-like receptors in pancreatic cancer. World J Gastroenterol 20: 5808-5817.

14. Morse DL, Balagurunathan Y, Hostetter G, et al. (2010): Identification of novel pancreatic adenocarcinoma cell-surface targets by gene expression profiling and tissue microarray. Biochem Pharmacol 80: 748-754.

15. Ochi A, Graffeo CS, Zambirinis CP, et al. (2012): Toll-like receptor 7 regulates pancreatic carcinogenesis in mice and humans. J Clin Invest 122: 4118-4129.

16. Ochi A, Nguyen AH, Bedrosian AS, et al. (2012): MyD88 inhibition amplifies dendritic cell capacity to promote pancreatic carcinogenesis via Th2 cells. J Exp Med 209: 1671-1687.

17. Drexler SK, Foxwell BM (2010): The role of toll-like receptors in chronic inflammation. Int J Biochem Cell Biol 42: 506-518.

18. Fischer M, Ehlers M (2008): Toll-like receptors in autoimmunity. Ann N Y Acad Sci 1143: 21-34.

19. Takeuchi O, Akira S (2010): Pattern recognition receptors and inflammation. Cell 140: 805-820.

20. Rakoff-Nahoum S, Medzhitov R (2009): Toll-like receptors and cancer. Nat Rev Cancer 9: 57-63.
21. Chung JY, Park YC, Ye H, Wu H (2002): All TRAFs are not created equal: common and distinct molecular mechanisms of TRAF-mediated signal transduction. J Cell Sci 115: 679-688.

22. Tseng PH, Matsuzawa A, Zhang W, et al. (2010): Different modes of ubiquitination of adaptor TRAF3 selectively activate the expression of type I interferons and proinflammatory cytokines. Nat Immunol 11: 70-75.

23. Park JS, Svetkauskaite D, He Q, et al. (2004): Involvement of toll-like receptors 2 and 4 in cellular activation by high mobility group box 1 protein. J Biol Chem 279: 7370-7377.

24. Bustin M (1999): Regulation of DNA-dependent activities by the functional motifs of the high-mobility-group chromosomal proteins. Mol Cell Biol 19: 5237-5246.

25. Wang H, Yang H, Czura CJ, et al. (2001): HMGB1 as a late mediator of lethal systemic inflammation. Am J Respir Crit Care Med 164: 1768-1773.

26. El Gazzar M (2007): HMGB1 modulates inflammatory responses in LPS-activated macrophages. Inflamm Res 56: 162-167.

27. Tsujimoto H, Ono S, Majima T, et al. (2005): Neutrophil elastase, MIP-2, and TLR-4 expression during human and experimental sepsis. Shock 23: 39-44.

28. Schaaf B, Luitjens K, Goldmann T, et al. (2009): Mortality in human sepsis is associated with downregulation of Toll-like receptor 2 and CD14 expression on blood monocytes. Diagn Pathol 4: 12-19.

29. Subramanian S, Tus K, Li QZ, et al. (2006): A Tlr7 translocation accelerates systemic autoimmunity in murine lupus. Proc Natl Acad Sci U S A 103: 9970-9975.

30. Roelofs MF, Wenink MH, Brentano F, et al. (2009): Type I interferons might form the link between Toll-like receptor (TLR) 3/7 and TLR4-mediated synovial inflammation in rheumatoid arthritis (RA). Ann Rheum Dis 68: 1486-1493.

31. Devaraj S, Dasu MR, Rockwood J, et al. (2008): Increased Toll-like receptor (TLR) 2 and TLR4 expression in monocytes from patients with type 1 diabetes: further evidence of a proinflammatory state. J Clin Endocrinol Metab 93: 578583.

32. Yu L, Chen S (2008): Toll-like receptors expressed in tumor cells: target for therapy. Cancer Immunol Immunother 57: 1271-1278.

33. Zhang JJ, Wu HS, Wang L, et al. (2010): Expression and significance of TLR4 and HIF- $1 \alpha$ in pancreatic ductal adenocarcinoma. World J Gastroenterol 16: 2881-2888.

34. Huang B, Zhao J, Li H, et al. (2005): Toll-like receptors on tumor cells facilitate evasion of immune surveillance. Cancer Res 65: 5009-5014.

35. Sato Y, Goto Y, Narita N, Hoon DS (2009): Cancer cells expressing Toll-like receptors and the tumor microenvironment. Cancer Microenviron 2: 205-214.

36. Zhou M, McFarland-Mancini MM, Funk HM, et al. (2009): Toll-like receptor expression in normal ovary and ovarian tumors. Cancer Immunol Immunother 58: 1375-1385.

37. Wang EL, Qian ZR, Nakasono M, et al. (2010): High expression of Toll-like receptor 4/myeloid differentiation factor 88 signals correlates with poor prognosis in colorectal cancer. Br J Cancer 102: 908-915.

38. Rakoff-Nahoum S, Medzhitov R (2009): Toll-like receptors and cancer. Nat Rev Cancer 9: 57-63.

39. Basith S, Manavalan B, Yoo TH, et al. (2012): Roles of tolllike receptors in cancer: a double-edged sword for defense and offense. Arch Pharm Res 35: 1297-1316. 
40. Wang JH, Manning BJ, Wu QD, et al. (2003): Endotoxin/ lipopolysaccharide activates NF- $\mathrm{KB}$ and enhances tumor cell adhesion and invasion through a $\beta-1$ integrin-dependent mechanism. J Immunol 170: 795-804.

41. Ikebe M, Kitaura Y, Nakamura M, et al. (2009): Lipopolysaccharide (LPS) increases the invasive ability of pancreatic cancer cells through the TLR4/MyD88 signaling pathway. J Surg Oncol 100: 725-773.

42. Onier N, Hilpert S, Arnould L, et al. (1999): Cure of colon cancer metastasis in rats with the new lipid A OM 174. Apoptosis of tumor cells and immunization of rats. Clin Exp Metastasis 17: 299-306.

43. Reisser D, Pance A, Jeannin JF (2002): Mechanisms of antitumoral effect of lipid A. Bioessays 24: 284-289.

44. Isambert N, Fumoleau P, Paul C, et al. (2013): Phase I study of OM-174, a lipid A analogue, with assessment of immunological response, in patients with refractory solid tumors. BMC Cancer 13: 172-182.

45. Zambirinis CP, Ochi A, Barilla R, et al. (2013): Induction of TRIF-or MYD88-dependent pathways perturbs cell cycle regulation in pancreatic cancer. Cell Cycle 12: 1153-1154.

46. Sun H, Li XB, Meng Y, et al. (2013): TRAF6 upregulates expression of HIF-1alpha and promotes tumor angiogenesis. Cancer Res 73: 4950-4959.

47. Carneiro LA, Travassos LH, Philpott DJ (2004): Innate immune recognition of microbes through Nod1 and Nod2: implications for disease. Microbes Infect 6: 609-616.

48. Sońdka Z, Tretyn A, Szeliga J, et al. (2006): Involvement of leucine rich repeats (LRR) domain containing proteins in molecular mechanisms of innate immunity of plants and animals. Post Biol Kom 33: 635-656.

49. Chamaillard M, Hashimoto M, Horie Y, et al. (2003): An essential role for Nod1 in host recognition of bacterial peptidoglykan containing diaminopimelic acid. Nat Immunol 4: 702-707.

50. Girardin SE, Boneca IG, Viala J, et al. (2003): Nod2 is a general sensor of peptidoglykan through muramyl dipeptide (MDP) detection. J Biol Chem 278: 8869-8872.

51. Lee JY, Hwang DH (2006): The modulation of inflammatory gene expression by lipids: mediation through Toll-like receptors. Mol Cells 21: 174-185.

52. Jiang W, Wang X, Zeng B, et al. (2013): Recognition of gut microbiota by NOD2 is essential for the homeostasis of intestinal intraepithelial lymphocytes. J Exp Med 210: 2465-2476.

53. Chen GY, Shaw MH, Redondo G, Núńez G (2008): The innate immune receptor Nod1 protects the intestine from inflammation-induced tumorigenesis. Cancer Res 68: 1006010067.

54. Companioni O, Bonet C, Muńoz X, et al. (2013): Polymorphisms of Helicobacter pylori signaling pathway genes an gastric cancer risk in the European prospective investigation into cancer-eurgast cohort. Int J Cancer 134: 92-101.

55. Livak KJ, Schmittgen TD (2001): Analysis of relative gene expression data using real-time quantitative PCR and 2(-Delta Delta C(T)) Method. Methods 25: 402-408.

56. Del Pozo JL (2010): Primers on molecular pathways: lipopolysaccharide signaling-potential role in pancreatitis and pancreatic cancer. Pancreatology 10: 114-118.

57. Vonlaufen A, Xu Z, Daniel B, et al. (2007): Bacterial endotoxin: a trigger factor for alcoholic pancreatitis? Evidence from a novel, physiologically relevant animal model. Gastroenterology 133: 1293-1303.
58. Visintin A, Mazzoni A, Spitzer JH, et al. (2001): Regulation of Toll-like receptors in human monocytes and dendritic cells. J Immunol 166: 249-255.

59. Li D, Duell EJ, Yu K, et al. (2012): Pathway analysis of genome-wide association study data highlights pancreatic development genes as susceptibility factors for pancreatic cancer. Carcinogenesis 33: 1384-1390.

60. Pérez-Bárcena J, Regueiro V, Crespí C, et al. (2010): Expression of toll-like receptors 2 and 4 is upregulated during hospital admission in traumatic patients: lack of correlation with blunted innate responses. Ann Surg 251: 521-527.

61. Härter L, Mica L, Stocker R, et al. (2004): Increased expression of Toll-like receptor- 2 and -4 on leukocytes from patients with sepsis. Shock 22: 403-409.

62. Armstrong L, Medford AR, Hunter KJ, et al. (2004): Differential expression of Toll-like receptor (TLR)-2 and TLR-4 on monocytes in human sepsis. Clin Exp Immunol 136: 312-319.

63. Takeuchi O, Takeda K, Hoshino K, et al. (2000): Cellular responses to bacterial cell wall components are mediated through MyD-88 dependent signaling cascades. Int Immunol 12: 113-117.

64. Weighardt H, Kaiser-Moore S, Vabulas RM, et al. (2002): Cutting edge: myeloid differentiation factor 88 deficiency improves resistance against sepsis causes by polymicrobial infection. J Immunol 169: 2823-2827.

65. Fan P, Zhang JJ, Wang B, et al. (2012): Hypoxia-inducible factor-1 upregulates the expression of Toll-like receptor 4 in pancreatic cancer cells under hypoxic conditions. Pancreatology 12: 170-178.

66. Kurahara H, Shinchi H, Mataki Y, et al. (2011): Significance of M2-polarized tumor-associated macrophage in pancreatic cancer. J Surg Res 167: 211-219.

67. Baran B, Bechyne I, Siedlar M, et al. (2009): Blood monocytes stimulate migration of human pancreatic carcinoma cells in vitro: the role of tumour necrosis factor-alpha. Eur J Cell Biol 88: 743-752.

68. Reid MD, Basturk O, Thirabanjasak D, et al. (2011): Tumor-infiltrating neutrophils in pancreatic neoplasia. Mod Pathol 24: 1612-1619.

69. Kędziora S, Słotwiński R, Dąbrowska A, et al. (2012): Glutamine abolishes the TLR4 gene overexpression in pancreatic cancer patients: a preliminary study. Centr Eur J Immunol 37: 350-354.

70. Kessel A, Toubi E, Pavlotzky E, et al. (2008): Treatment with glutamine is associated with down-regulation of Toll-like receptor- 4 and myeloid differentiation factor 88 expression and decrease in intestinal mucosal injury caused by lipopolysaccharide endotoxemia in a rat. Clin Exp Immunol 151: 341347.

71. Lee JY, Plakidas A, Lee WH, et al. (2003): Differential modulation of Toll-like receptors by fatty acids: preferential inhibition by $n-3$ polyunsaturated fatty acids. J Lipid Res 44 : 479-486.

72. Molteni M, Marabella D, Orlandi C, Rossetti C (2006): Melanoma cell lines are responsive in vitro to lipopolysaccharide and express TLR-4. Cancer Lett 235: 75-83.

73. Chen K, Huang J, Gong W, et al. (2007): Toll-like receptors in inflammation, infection and cancer. Int Immunopharmacol 7: 1271-1285

74. Starczynowski DT, Lockwood WW, Deléhouzée S, et al. (2014): TRAF6 is an amplified Oncogene bridging the RAS and NF- $\kappa B$ pathways in human lung cancer. J Clin Invest 121: 4095-4105. 
75. Vaz J, Akbarshahi H, Andersson R (2013): Controversial role of toll-like receptors in acute pancreatitis. World J Gastroenterol 19: 616-630.

76. Schwartz AL, Malgor R, Dickerson E, et al. (2009): Phenylmethimazone decreases Toll-like receptor 3 and noncanominal Wnt5a expression in pancreatic cancer and melanoma together with tumor cell growth and migration. Clin Cancer Res 15: 4114-4122.

77. Kemp TJ, Ludwig AT, Earel JK, et al. (2005): Neutrophil stimulation with Mycobacterium bovis bacillus Calmette-Guerin (BCG) results in release of functional TRAIL/Apo-2L. Blood 106: 3474-3482.

78. Manegold C, Gravenor D, Woytowitz D, et al. (2008): Randomized phase II trial of a toll-like receptor 9 agonist oligodeoxynucleotide, PF-3512676, in combination with firstline taxane plus platinum chemotherapy for advanced-stage non-small-cell lung cancer. J Clin Oncol 26: 3979-3986.

79. Mauri D, Kamposioras K, Tsali L, et al. (2010): Overall survival benefit for weekly vs.three-weekly taxanes regimens in advanced brest cancer: A meta-analysis. Cancer Treat Rev 36: 69-74.

80. Garay RP, Viens P, Bauer J, et al. (2007): Cancer relapse under chemotherapy: Why TLR2/4 receptor agonists can help. Eur J Pharmacol 563: 1-17.

81. Adams S (2009): Toll-like receptor agonists in cancer therapy. Immunotherapy 1: 949-964.

82. Chung HW, Lim JB, Jang S, et al. (2012): Serum high mobility group box-1 is a powerful diagnostic and prognostic biomarker for pancreatic ductal adenocarcinoma. Cancer Sci 103: 1714-1721.

83. Kang R, Tang D, Schapiro NE, et al. (2010): The receptor for advanced glycation end products (RAGE) sustains autophagy and limits apoptosis, promoting pancreatic tumor cell survival. Cell Death Differ 17: 666-676.

84. Chung HW, Lee SG, Kim H, et al. (2009): Serum high mobility group box-1 (HMGB1) is closely associated with the clinical and pathologic features of gastric cancer. J Transl Med 7: 38-49.

85. Kang R, Tang D, Schapiro NE, et al. (2014): The HMGB1/ RAGE inflammatory pathway promotes pancreatic tumor growth by regulating mitochondrial bioenergetics. Oncogene 33: 567-577. 